\title{
Measurement of diffusion coefficients by radioanalysis
}

\author{
H. A. Das \\ ECN, P.O. Box 1, 1755 ZG Petten (N. H.), The Netherlands
}

(Received June 27, 2006)

Radioanalysis, more particularly the use of radiotracers, enables measurement of coefficients of mass- and self-diffusion by simple benchtop experiments. They are related to environmental inquiries and materials science mainly. Current procedures are surveyed here.

\section{Introduction}

Diffusion is an ubiquitous phenomenon in both environmental investigations and materials science. Determination of diffusion coefficients in a variety of systems is mandatory to the quantification of masstransport of toxic or essential constituents and thus to decision making. Self diffusion and isotopic exchange in solids are keys to the understanding of atomic mobility in metals.

Diffusion in a single phase is met in gases $(G)$ and solutions (L). That between two adjacent phases includes gas-liquid (G-L) gas-solid (G-S), liquid-solid (L-S) and solid-solid ( S-S ) combinations. In environmental management G-L, G-S and L-S are the most important cases. Measurements in materials science deal with solid systems mainly.

The scope of radioanalysis in determining the pertaining diffusion coefficients is surveyed here. References $1-10$ provide the principles while our texts 11-20 are available upon request.

\section{Diffusion of gases into a liquid with and without chemical reaction $(G-L)$}

Absorption of gases in a liquid is a standard operation in chemical technology. Purification of an airstream by absorption of noxious traces is an example of obvious environmental importance. Combination of absorption with chemical reaction in the liquid enhances the efficacy. In the case of a trace constituent this implies a (pseudo-) first order reaction.

The absorbent may be kept homogeneous, e.g., by continuous stirring, or just stagnant. In the first case absorption is governed by a transfer parameter, expressed in $\mathrm{cm} \cdot \mathrm{s}^{-1}$. The alternative implies a diffusion coefficient of dimension $\mathrm{cm}^{2} \cdot \mathrm{s}^{-1}$. Both formulations rely on a Henry-constant which relates the concentrations in gas- and liquid-phase at equilibrium.

The use of molecular radiotracers or radiolabeled compounds enables an efficacious and sensitive experimental procedure for the observation of time- dependent absorption under batch conditions. A special case is met in ${ }^{222} \mathrm{Rn} \quad\left(T_{1 / 2}=3.85 \mathrm{~d}\right)$ where decay mimicks a first-order reaction with the absorbent. Pertaining formulation and experimental conditions are given in Reference 11. In laboratory experiments, a ${ }^{226} \mathrm{Ra}$ source in a $<50 \mathrm{~cm}^{3}$ beaker with $\sim 20 \mathrm{~cm}^{3}$ overhead space and closed with a removable lid is placed, together with a $<200 \mathrm{~cm}^{3}$ beaker, again with a removable lid, containing water as the absorbent in a 2liter box which can be sealed hermetically. Both liquid volumes can be stirred while the air is circulated in closed circuit by a peristaltic pump. The increase with time of the specific activity of the absorbent yields the transport parameter. It is measured batchwise; continuous counting by way of a closed loop is possible.

The source is calibrated by quantitative collection of (1) ${ }^{222} \mathrm{Rn}$ on a cartridge with $\sim 80 \mathrm{~g}$ active carbon and absolute counting of the daughter products ${ }^{214} \mathrm{~Pb}$, $\left(T_{1 / 2}=26.8 \mathrm{~min}\right)$, and (2) ${ }^{214} \mathrm{Bi}, \quad\left(T_{1 / 2}=19.8 \mathrm{~min}\right)$. Details on collection and counting are given in Reference 12.

\section{Emanation of ${ }^{222} R n$ from solids $(G-S)$}

Measurement of ${ }^{222} \mathrm{Rn}$ emanating from building components is now legally required in the EU. This implies two preparatory actions: (1) formulation in terms of the governing parameters, the ${ }^{226} \mathrm{Ra}$ content and the effective diffusion coefficient, and (2) design of a routine procedure for specimens of simple geometrical shape.

Three approaches are of practical interest: one dimensional diffusion from "infinitely" thick and from thin slabs, and three-dimensional diffusion from either blocks or discs. Both the ${ }^{226} \mathrm{Ra}$ content and the ${ }^{222} \mathrm{Rn}$ diffusion coefficient can be obtained by gamma-ray spectrometry of the ${ }^{222} \mathrm{Rn}$ progeny. Details on formulation and procedures are available in Reference 13. Measurements on granular solids are influenced by grain shape and -size, and their inherent spread, as formulated in Reference 14. 
In both cases a $100 \mathrm{~cm}^{3}$ HPGe detector, of $3^{\prime \prime}=7.5 \mathrm{~cm}$ diameter, with an $8 \mathrm{k}$ MCA at $0.25 \mathrm{keV} /$ channel is used to measure the $352 \mathrm{keV} \gamma$-ray of ${ }^{214} \mathrm{~Pb}$ and the $609 \mathrm{keV}$ of ${ }^{214} \mathrm{Bi}$.

\section{Measurement of diffusion coefficients in granular materials $(L-S)$}

Diffusion of trace constituents through wet granular material is an obvious environmental concern. Assessment of the effective diffusion coefficient as a function of the water content is necessary to predict the future spread of pollution.

Interpretation should be based on the distinction between the coefficients of self- and mass-diffusion, $D_{s}$ and $D_{m}$. If both processes occur simultaneously, they combine into one apparent diffusion coefficient, $D_{a}$. If isotope effects can be neglected, which is usual for $Z$ values $>10$ (Reference 8 ), $D_{s}$ is equal to the diffusion coefficient of isotopic exchange. Equally, at very low concentrations $D_{m}$ approaches $D_{s}$. Both $D_{s}$ and $D_{m}$ are related to their corresponding value in dilute solution, $D_{0}$, by:

$$
D=\left(D_{0} / r\right) /\left(1+m_{s} / m_{l}\right)
$$

with $r$ is the physical restriction factor, usually called tortuosity, and $m_{s} / m_{l}$ is the local distribution ratio. ${ }^{15}$

The determination of $D_{s}$ and $D_{m}$ in granular materials is described in detail in References 15-17. Practice is summarized in Fig. 1. Carrier-free ${ }^{170} \mathrm{Tm}$ serves as the boundary marker.

$D_{s}$ : Of two originally identical aliquots, one is spiked with a, preferably carrier-free radionuclide. They are then brought into contact within a small tube of $1 \mathrm{~cm}^{2}$ cross section and $25-30 \mathrm{~mm}$ length per aliquot-section. After 7-20 days the total sample is sliced into $1 \mathrm{~mm}$ sections which are counted under identical conditions. The experimental range is from $\sim 10^{-5}$ to $\sim 10^{-10} \mathrm{~cm}^{2} \cdot \mathrm{s}^{-1}$ with a precision of $5-10 \%$.

$D_{m}$ : The concentration in one of two originally identical aliquots is doubled and a radiotracer spike is added, after which the same procedure follows. The originally available concentration of the trace compound involved is determined by separate leaching experiments reported in Reference 18.

The ratio surface-bound/pore-water becomes of influence at low water content. ${ }^{16}$ Figure 2 gives the variation of the restriction factor, $r$, as a function of the water-content for a basic fly-ash, as measured with the inert (i.e., for which $m_{s} / m_{l}=0$ ) radiotracer ${ }^{99} \mathrm{MoO}_{4}$ ".

Corresponding measurements are performed with porous ceramics. ${ }^{17}$

$D_{s}$ : A completely wetted test specimen of known effective porosity, is allowed to come into equilibrium with a solution of known concentration and volume. A radioactive spike is added at time zero. Equation (2) applies

$$
\frac{\text { Vol. L }}{\text { intern. vol. S }} \cdot\left[\frac{(\text { spec. act. } \mathrm{L})_{0}}{(\text { spec. act. } \mathrm{L})_{t}}-1\right]=f(D, t)
$$

The function is discussed in Reference 1 and shown here as Fig. 3 for $D$-values from $10^{-5}$ to $10^{-9} \mathrm{~cm}^{2} \cdot \mathrm{s}^{-1}$.

$D_{m}$ : Now the water-saturated test specimen is placed in a radiolabeled solution of known volume and concentration. Equation (2) is used again.

Application of a radiotracer solution to assess the effective porosity is a related experiment. In case of granular material shape and size distribution have to be taken into account as formulated in Reference 19.

\section{Diffusion coefficients in pure metals and binary alloys $(L-S)$}

Measurement of the (self-) diffusion in metals is one of the oldest uses of radiotracers. Experiments are performed with small cylindrical bars, usually at elevated temperatures. Self-diffusion coefficients in the pure metals $\mathrm{A}$ and $\mathrm{B}$ are indicated as $D_{\mathrm{AA}}$ and $D_{\mathrm{BB}}$. Their determination by radiotracers is based on the activity profile after some appropriate time of contact between two identical aliquots, one of which has been spiked. Alternatively, just one bar is covered at one end with a very thin layer of radiotracer. Interpretation is based on Fick's second law with a constant diffusion coefficient. This is known as the GRUBE procedure. ${ }^{5}$

Mass diffusion coefficients of $\mathrm{A}$ and $\mathrm{B}$ in binary alloys of various composition are known as the intrinsic coefficients, $D_{\mathrm{A}}$ and $D_{\mathrm{B}}$. They combine into the interdiffusion coefficient $D_{\mathrm{AB}}$, according to the first of Darken's equations:

$$
D_{\mathrm{AB}}=N_{\mathrm{A}} \cdot D_{\mathrm{A}}+N_{\mathrm{B}} \cdot D_{\mathrm{B}}
$$

in which $N_{\mathrm{A}}$ and $N_{\mathrm{B}}$ are the atomic volume fractions.

Recording of the concentration profile and the rate of displacement of the original border-plane between the two sections (Kirkendall-effect, $v$ in $\mathrm{cm}^{-1} \mathrm{~s}^{-1}$ ) may be done by any analytical method, including NAA. Its resolution into the position dependent $D_{\mathrm{AB}}$-values is again by Fick's second law, now with a varying diffusion coefficient according to the MATANO procedure. $^{5}$

Darken's second equation connects the Kirkendalleffect to the local values of $D_{\mathrm{A}}$ and $D_{\mathrm{B}}$ and composition:

$$
v=\left(D_{\mathrm{A}}-D_{\mathrm{B}}\right) \cdot \delta N_{\mathrm{A}} / \delta x
$$

Combination of Eqs (3) and (4) then yields $D_{\mathrm{A}}$ and $D_{\mathrm{B}}$. The ratio:

$$
\left(D_{\mathrm{A}}\right)_{m} /\left(D_{\mathrm{A}}\right)_{s}=\left(D_{\mathrm{B}}\right)_{m} /\left(D_{\mathrm{B}}\right)_{s}
$$

indicates the deviation from thermodynamic ideality. 

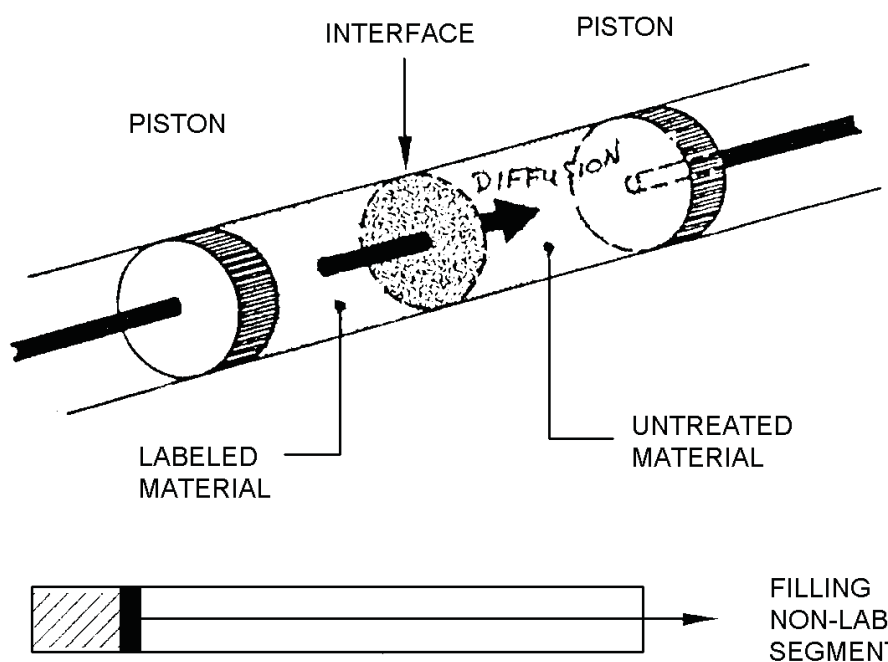

FILLING

NON-LABELED

SEGMENT

$\stackrel{T m-170}{\longrightarrow}$

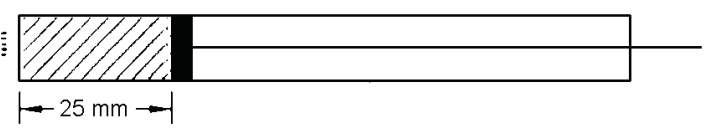

INTERFACE

MARKER

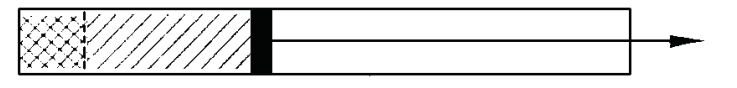

FILLING

LABELED

SEGMENT
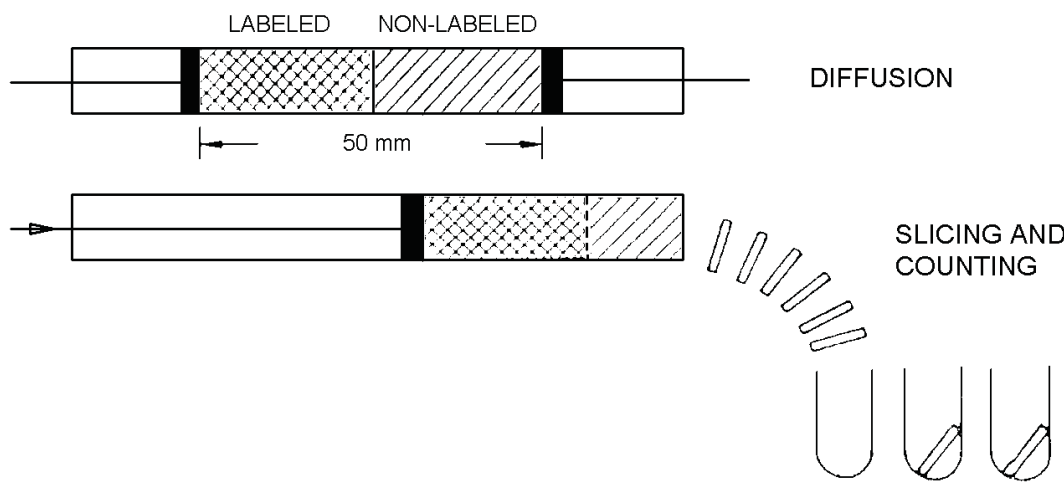

Fig. 1. Miniaturized measurement of diffusion in granular materials

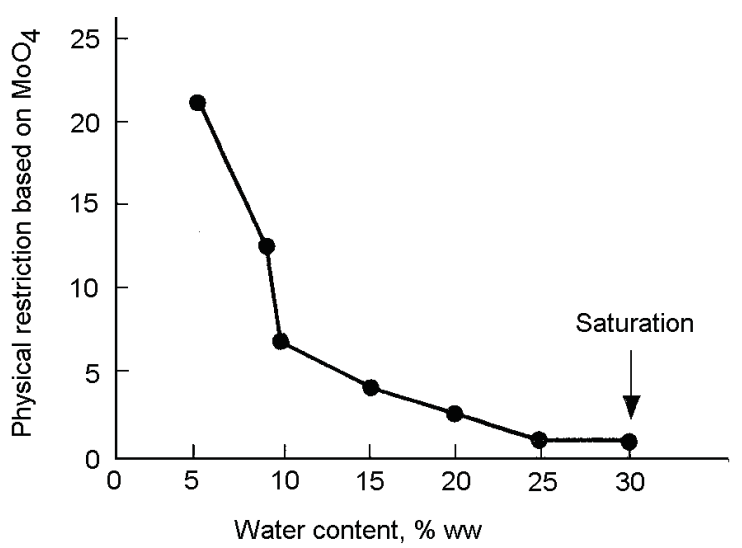

Fig. 2. Variation of the physical restriction factor with the water-content 


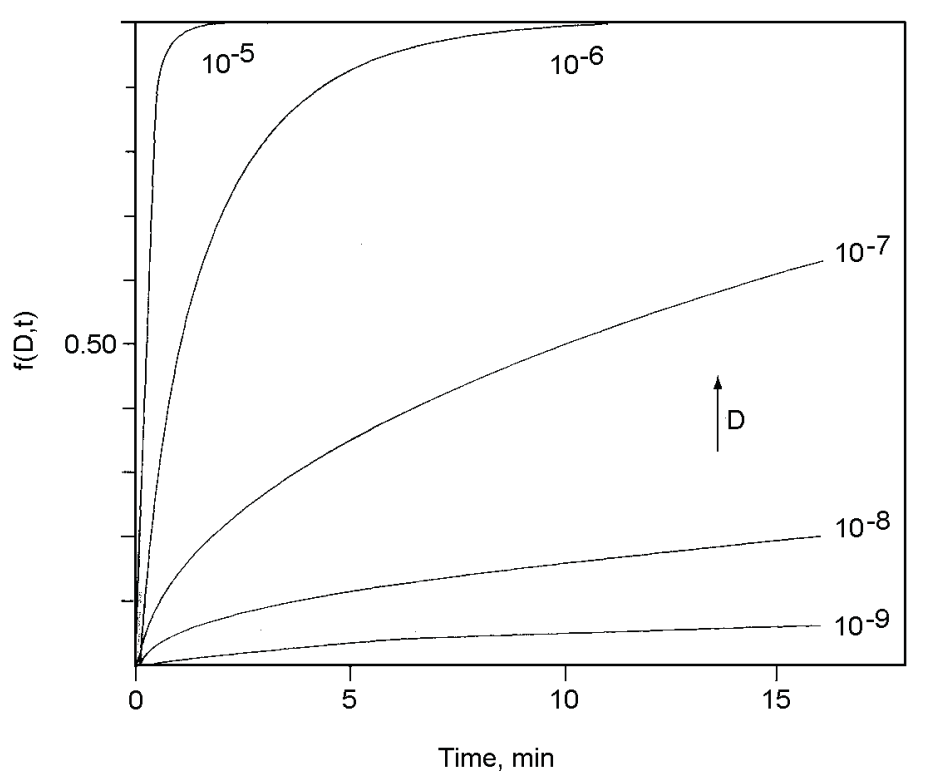

Fig. 3. The function $f(D, t)$ from Eq. (2) as a function of time in days for $10^{-9}<D<10^{-5} \mathrm{~cm}^{2} \cdot \mathrm{s}^{-1}$

\section{Mass diffusion in a single solid phase (S)}

An example is met in the diffusion of $\mathrm{P}$ in oligocrystalline $\mathrm{Si}$, encountered in the production of solar cells. Diffusion occurs along the grain boundaries and through the crystals. High precision is required to separate these two phenomena. This can be achieved with ${ }^{32} \mathrm{P}, T_{1 / 2}=14.3 \mathrm{~d}$, as radiotracer. Details are given in Reference 20.

First cleaned with acetone, $2 \mathrm{~N} \mathrm{HNO}_{3}$, cone. $\mathrm{HF}$ and water, cylindrically shaped samples are cut and mirrorpolished. Then they are annealed in silica ampoules together with a droplet of $\mathrm{H}_{3} \mathrm{PO}_{4}$ with $7.4 \mathrm{~Bq}(200 \mathrm{mCi})$ ${ }^{32} \mathrm{P}$ per mmol. After diffusion and annealing, a layer of $50-100 \mu \mathrm{m}$ is ground off from the cylindrical surface. From both flat sides layers of $>1 \mu \mathrm{m}$ are polished off with a copper disk and diamond paste, using a precision polishing apparatus developed at Philips Research Laboratories. Their masses are obtained by differential weighing with a microbalance at $\pm 10 \mu \mathrm{g}$, corresponding to $\sim 10 \%$ precision.

Table 1 gives the result of a comparison between the data from Cerenkov counting of the collected material at one hand and differential solid scintillation counting on the remaining sample. The latter procedure can be used as a quasi-exponential profile of beta-activity is involved.

\begin{tabular}{|c|c|c|}
\hline \multirow{2}{*}{$\begin{array}{c}\text { Depth, } \\
\mu \mathrm{m}\end{array}$} & \multicolumn{2}{|c|}{ Concentration in relative unites } \\
\hline & From LS & From SS \\
\hline 0.31 & $10.50 \pm 0.09$ & $10.50 \pm 0.09$ \\
\hline 0.83 & $10.52 \pm 0.14$ & $10.57 \pm 0.14$ \\
\hline 1.42 & $10.45 \pm 0.08$ & $10.45 \pm 0.08$ \\
\hline 2.16 & $9.80 \pm 0.09$ & $9.79 \pm 0.09$ \\
\hline 2.74 & $9.89 \pm 0.12$ & $9.95 \pm 0.12$ \\
\hline 3.57 & $9.59 \pm 0.05$ & $9.60 \pm 0.05$ \\
\hline 4.62 & $9.11 \pm 0.07$ & $9.13 \pm 0.07$ \\
\hline 5.95 & $8.72 \pm 0.04$ & $8.74 \pm 0.04$ \\
\hline 7.19 & $7.86 \pm 0.08$ & $7.80 \pm 0.08$ \\
\hline 8.14 & $6.12 \pm 0.09$ & $6.22 \pm 0.07$ \\
\hline 9.35 & $5.19 \pm 0.13$ & $5.33 \pm 0.08$ \\
\hline 11.07 & $4.56 \pm 0.16$ & $4.44 \pm 0.08$ \\
\hline 13.25 & $2.50 \pm 1.0$ & $3.1 \pm 0.3$ \\
\hline 15.4 & $0.7 \pm 1.80$ & $1.3 \pm 1.7$ \\
\hline
\end{tabular}

\section{Conclusions}

Radioanalysis is a valuable and often indispensable tool in the study of diffusion phenomena in both environmental investigations and materials science, once the proper formulation of the intended application has been obtained. It depends on the further existence of nuclear centers and radionuclide laboratories at industries and universities. 


\section{References}

1. J. CRANK, The Mathematics of Diffusion, 2nd ed., Clarendon Press, Oxford, 1975.

2. TH. K. Sherwood, R. L. Pigford, Ch. R. Wilke, Mass Transfer, McGraw-Hill/Kogakusha Ltd., Tokyo, 1975.

3. J. BEAR, Dynamics of Fluids in Porous Media, Dover Publications Inc., New York, 1988.

4. J. B. Bird, W. E. Stewart, E. N. Lightfoot, Transport Phenomena, Wiley International, New York, 1988.

5. R. E. ReED-Hill, R. ABbaschian, Physical Metallurgy Principles, 3rd ed., PWS Publishing Co., Boston, 1991.

6. E. L. Cussler, Diffusion, Mass Transfer in Fluid Systems, 2nd ed., Cambridge University Press, 1997.

7. C. Eeymonie, Les traceurs radioactifs en métallurgie physique, Dunod, Paris, 1960.

8. L. MELander, Isotope Effects in Reaction Rates, The Ronald Press Company, New York, 1990.

9. C. W. ShePPARD, Basic Principles of the Tracer Method, J. Wiley and Sons, New York, 1962.
10. H. J. M. Bowen, Chemical Applications of Radiotracers, Methuen Co. Ltd., London, ECN publications and informal reports, 1969.

11. Measurement of the absorption of trace gases in water with and without reaction as exemplified by ${ }^{222} \mathrm{Rn}$, May 2004.

12. Sampling of gaseous trace constituents from air, February 2002.

13. Emanation of ${ }^{222} \mathrm{Rn}$ from solid bodies of building materials and its measurement, November 2002.

14. Emanation of ${ }^{222} \mathrm{Rn}$ from granular material, February 2004.

15. H. A. DAs, H. A. VAN DER Shoot, J. WiJKstra, Appl. Radiation Isotopes, 40 (1989) 615.

16. H. A. DAS, Appl. Radiation Isotopes, 44 (1993) 1245.

17. Radioanalysis in materials science, September 2001.

18. Leaching of trace constituents from inorganic granular wastes. Formulation, practical limitations and use of radiotracers, January 2003.

19. Diffusion coefficient and porosity of granular material, Determination by radiotracer measurements, March 2004.

20. F. H. M. SPIT, H. A. DAS, Isotopenpraxis, 24 (1988) 56. 